\title{
Front Matter: Volume 7122
}

, "Front Matter: Volume 7122," Proc. SPIE 7122, Photomask Technology 2008, 712201 (25 November 2008); doi: 10.1117/12.817633

SDIE Event: SPIE Photomask Technology, 2008, Monterey, California, United SPIE. States 


\title{
Photomask Technology 2008
}

\author{
Hiroichi Kawahira \\ Larry S. Zurbrick \\ Editors
}

7-10 October 2008

Monterey, California, USA

Sponsored by

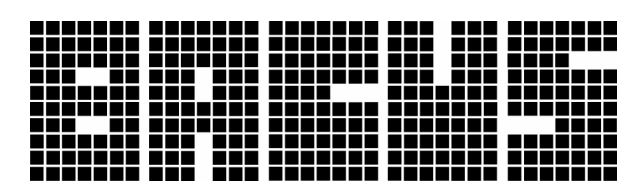

The international technical group of SPIE dedicated

to the advancement of photomask technology

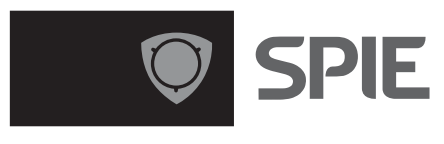

Published by

SPIE

Volume 7122

Part One of Two Parts

Proceedings of SPIE, 0277-786X, v. 7122

SPIE is an international society advancing an interdisciplinary approach to the science and application of light. 
The papers included in this volume were part of the technical conference cited on the cover and title page. Papers were selected and subject to review by the editors and conference program committee. Some conference presentations may not be available for publication. The papers published in these proceedings reflect the work and thoughts of the authors and are published herein as submitted. The publisher is not responsible for the validity of the information or for any outcomes resulting from reliance thereon.

Please use the following format to cite material from this book:

Author(s), "Title of Paper," in Photomask Technology 2008, edited by Hiroichi Kawahira, Larry S. Zurbrick, Proceedings of SPIE Vol. 7122 (SPIE, Bellingham, WA, 2008) Article CID Number.

ISSN 0277-786X

ISBN 9780819473554

Published by

SPIE

P.O. Box 10, Bellingham, Washington 98227-0010 USA

Telephone +1 3606763290 (Pacific Time) · Fax +1 3606471445

SPIE.org

Copyright (C 2008, Society of Photo-Optical Instrumentation Engineers

Copying of material in this book for internal or personal use, or for the internal or personal use of specific clients, beyond the fair use provisions granted by the U.S. Copyright Law is authorized by SPIE subject to payment of copying fees. The Transactional Reporting Service base fee for this volume is $\$ 18.00$ per article (or portion thereof), which should be paid directly to the Copyright Clearance Center (CCC), 222 Rosewood Drive, Danvers, MA 01923. Payment may also be made electronically through CCC Online at copyright.com. Other copying for republication, resale, advertising or promotion, or any form of systematic or multiple reproduction of any material in this book is prohibited except with permission in writing from the publisher. The CCC fee code is $0277-786 \mathrm{X} / 08 / \$ 18.00$.

Printed in the United States of America.

Publication of record for individual papers is online in the SPIE Digital Library.

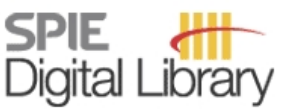

SPIEDigitalLibrary.org

Paper Numbering: Proceedings of SPIE follow an e-First publication model, with papers published first online and then in print and on CD-ROM. Papers are published as they are submitted and meet publication criteria. A unique, consistent, permanent citation identifier (CID) number is assigned to each article at the time of the first publication. Utilization of CIDs allows articles to be fully citable as soon they are published online, and connects the same identifier to all online, print, and electronic versions of the publication. SPIE uses a six-digit CID article numbering system in which:

- The first four digits correspond to the SPIE volume number.

- The last two digits indicate publication order within the volume using a Base 36 numbering system employing both numerals and letters. These two-number sets start with 00, 01, 02, 03, 04, 05, $06,07,08,09,0 A, 0 B \ldots 0 Z$, followed by $10-12,20-2 Z$, etc.

The CID number appears on each page of the manuscript. The complete citation is used on the first page, and an abbreviated version on subsequent pages. Numbers in the index correspond to the last two digits of the six-digit CID number. 


\title{
Contents
}

\section{Part One}

\author{
xix Conference Committee \\ xxiii Introduction \\ xxv Schedule of Special Section on Mask Infrastructure Challenges at 32nm and Beyond \\ xxvii Summary of Special Section on Mask Infrastructure Challenges at 32nm and Beyond
}

\section{PLENARY PAPER FROM ADVANCED LITHOGRAPHY 2008}

712202 Lithography and design in partnership: a new roadmap (AL08 Plenary Paper) [7122-500]

A. B. Kahng, Univ. of California, San Diego (United States)

\section{SESSION 1 INVITED SESSION}

712204 Mask industry assessment: 2008 [7122-02]

G. Hughes, SEMATECH (United States); H. Yun, SEMATECH (United States) and Intel Corp. (United States)

712207 PMJ panel discussion overview on mask complexities, cost, and cycle time in 32-nm system LSI generation: conflict or concurrent? [7122-05]

K. Hosono, Renesas Technology Corp. (Japan); K. Kato, SII NanoTechnology, Inc. (Japan)

\section{SESSION 2 MASK MATERIALS FOR OPTICAL EXTENSIONS}

712209 Characterization of binary and attenuated phase shift mask blanks for $32 \mathrm{~nm}$ mask fabrication [7122-07]

T. Faure, E. Gallagher, M. Hibbs, L. Kindt, K. Racette, R. Wistrom, A. Zweber, A. Wagner, IBM Corp. (United States); Y. Kikuchi, T. Komizo, S. Nemoto, Toppan Photomasks, Inc. (United States)

7122 OA Impact of the OMOG substrate on $32 \mathrm{~nm}$ mask OPC inspectability, defect sensitivity, and mask design rule restrictions [7122-08]

K. Badger, IBM Systems and Technology Group (United States); Y. Kodera, Toppan Photomasks, Inc. (United States); E. Gallagher, M. Lawliss, IBM Systems and Technology Group (United States)

$7122 \mathrm{OB} \quad$ Evaluation of 32nm advanced immersion lithography pellicles [7122-09]

N. Zhou, K. Racette, M. Hibbs, IBM Systems and Technology Group (United States); T. Mizoguchi, Toppan Photomasks, Inc. (United States); D. Hasselbeck, Michigan State Univ. (United States); M. Barrett, R. Nolan, IBM Systems and Technology Group (United States); F. Houle, IBM Almaden Research Ctr. (United States); J. Ritter, IBM Systems and Technology Group (United States); A. Wagner, IBM Advanced Lithography Research (United States); M. Caterer, IBM Systems and Technology Group (United States) 
7122 OC Road to a zero degree total temperature range post exposure bake process [7122-10] T. Wähler, P. Dress, HamaTech APE GmbH \& Co. KG (Germany)

7122 OD Study of second-generation Proximity Gap Suction Development System (PGSD-II) for mask fabrication [7122-11]

H. Sakurai, M. Terayama, M. Sakai, M. Itoh, O. Ikenaga, Toshiba Corp. (Japan);

H. Funakoshi, N. Sato, K. Nakamizo, M. Nomura, Tokyo Electron Kyushu, Ltd. (Japan): Y. Saito, Tokyo Electron, Ltd. (Japan); J. Nakao, Omron Corp. (Japan); N. Hayashi, Dai Nippon Printing Co., Ltd. (Japan)

7122 OE Process control of chrome dry etching by complete characterization of the RF power delivery [7122-12]

B. Sass, R. Schubert, T. Jakubski, S. Mauermann, P. Nesladek, A. Wiswesser, Advanced Mask Technology Ctr. GmbH \& Co. KG (Germany); K.-H. Gindra, SEMEQ Technologies GmbH (Germany); R. Malone, Pivotal Systems (United States)

$7122 \mathrm{OF} \quad$ Integrating $\mathrm{Cr}$ and MoSi etch for optimal photomask critical dimension uniformity and phase uniformity [7122-13]

R. Wistrom, IBM System and Technology Group (United States); T. Komizo, S. Nemoto, Toppan Photomasks, Inc. (United States); A. G. Reid, IBM System and Technology Group (United States)

$71220 \mathrm{E}$ Electric field-induced progressive CD degradation in reticles [7122-14]

G. C. Rider, Microtome (United States)

$7122 \mathrm{OH} \quad$ Don't kill canaries! Introducing a new test device to assess the electrostatic risk potential to photomasks [7122-15]

T. Sebald, ESTION GmbH \& Co. KG (Germany)

\section{SESSION 4 PATTERNING TECHNOLOGIES AND TOOLS}

7122 Ol Damage mechanisms and process optimization for photomasks with sub-resolution assist features [7122-16]

L. Kindt, E. Gallagher, J. Levin, IBM (United States); Y. Kodera, Toppan Photomasks, Inc. (United States); Y. Okawa, Y. Sasaki, Toppan Printing Co., Ltd. (Japan)

$71220 \mathrm{~J} \quad$ New electron optics for mask writer EBM-7000 to challenge hp 32nm generation [7122-17] T. Kamikubo, NuFlare Technology, Inc. (Japan); S. Golladay, R. Kendall, V. Katsap, NuFlare Technology USA (United States); K. Ohtoshi, NuFlare Technology, Inc. (Japan);

M. Ogasawara, S. Nishimura, Toshiba Corp. (Japan); R. Nishimura, O. lizuka, T. Nakayama, S. Shinkawa, T. Nishiyama, S. Tamamushi, NuFlare Technology, Inc. (Japan)

7122 OK E-beam exposure system using multi column cell (MCC) with CP for mask writing [7122-18] A. Yamada, H. Yasuda, M. Yamabe, Association of Super-Advanced Electronics Technologies (Japan) 
$7122 \mathrm{OL}$ Results obtained with the CHARPAN Engineering Tool and prospects of the ion Mask Exposure Tool (iMET) (Second Place Best Paper Award) [7122-19]

E. Platzgummer, H. Loeschner, G. Gross, IMS Nanofabrication AG (Austria)

$71220 \mathrm{M}$ Effects of heated substrates on bimetallic thermal resist for lithography and grayscale photomask applications [7122-20]

J. M. Dykes, P. Tsui, J. Leung, G. H. Chapman, Simon Fraser Univ. (Canada)

SESSION 5

DPL IMPLEMENTATION I

7122 ON Revisiting the layout decomposition problem for double patterning lithography (Invited Paper) [7122-21]

A. B. Kahng, C.-H. Park, Univ. of California, San Diego (United States); X. Xu, Magma Design Automation (United States); H. Yao, Univ. of California, San Diego (United States)

712200 Evaluation of Double Process Lithography (DPL) with bi-layer photo-resist process for contact layer-patterning [7122-22]

G. Chen, K. Wu, Freescale Semiconductor, Inc. (United States)

$7122 \mathrm{OP} \quad$ Mask defect printability in the spacer patterning process [7122-23]

S. Miyoshi, S. Yamaguchi, T. Hirano, H. Mashita, H. Mukai, A. Kobiki, Y. Kobayashi,

K. Hashimoto, S. Inove, Toshiba Corp. (Japan)

\section{SESSION 6 DPL IMPLEMENTATION II}

$71220 Q \quad$ Printability verification for double-patterning technology [7122-24]

G. Luk-Pat, Synopsys (United States); P. Panaite, Synopsys (Canada); K. Lucas, Synopsys

(United States); C. Cork, Synopsys SARL (France); V. Wiaux, S. Verhaegen, M. Maenhoudt, IMEC (Belgium)

7122 OR Double dipole RET investigation for $\mathbf{3 2} \mathbf{~ n m}$ metal layers [7122-25]

C. Babcock, Y. Zou, Advanced Micro Devices, Inc. (United States); D. Dunn, Z. Baum, Z. Zhao, IBM Microelectronics Division (United States); I. Matthew, Advanced Micro

Devices, Inc. (United States); P. LaCour, Mentor Graphics Corp. (United States)

7122 OS Double-patterning decomposition, design compliance, and verification algorithms at 32nm hp [7122-26]

A. Tritchkov, P. Glotov, S. Komirenko, E. Sahouria, A. Torres, A. Seoud, Mentor Graphics

Corp. (United States); V. Wiaux, IMEC (Belgium)

\section{SESSION 7 RET AND OPC/ORC I}

7122 OT Pixel-based SRAF implementation for 32nm lithography process [7122-27]

B.-S. Kim, Y.-H. Kim, S.-H. Lee, S.-I. Kim, S.-R. Ha, SAMSUNG Electronics Co., Ltd. (Korea,

Republic of); J. Kim, A. Tritchkov, Mentor Graphics (United States)

$7122 \mathrm{OU}$ Model-based assist feature placement: an inverse imaging approach [7122-28]

A. Poonawala, B. Painter, J. Mayhew, Synopsys, Inc. (United States) 
$7122 \mathrm{OV}$ Optimizing models based OPC fragmentation using genetic algorithms [7122-30] D. A. Dipaola, I. Stobert, IBM Semiconductor Research and Development Ctr. (United States)

$7122 \mathrm{OW} \quad$ Single exposure is still alive: gate patterning at $\mathbf{4 5 n m}$ technology node [7122-31] K. Herold, Infineon Technologies NA Corp. (United States); D. J. Samuels, D. Dunn, A. Abdo, IBM Systems and Technology Group (United States); C. Sarma, Infineon Technologies NA Corp. (United States)

7122 OX Advanced mask technique to improve bit line CD uniformity of $90 \mathrm{~nm}$ node flash memory in low-k1 lithography [7122-32]

J. Kim, J. Choi, J. Kim, J. Han, Dongbu HiTek (Korea, Republic of)

7122 OY OPC hotspot identification challenges: ORC vs. PWQ on wafer [7122-151]

A. Poock, S. McGowan, F. Weisbuch, AMD, Saxony LLC \& Co. KG (Germany);

G. Schnasse, R. Ghaskadvi, KLA-Tencor Corp. (United States)

$71220 \mathrm{Z}$ Challenges for the quality control of assist features for $45 \mathrm{~nm}$ node technology and beyond [7122-34]

L. Wang, T. Lukanc, M. Takahashi, H.-E. Kim, K. Phan, Spansion LLC (United States); T. Yamazaki, Y. Kojima, W. Nozaki, T. Haraguchi, Y. Okuda, Toppan Printing Co., Ltd. (Japan)

\section{SESSION 8 ADVANCED CLEANING}

712210 Impact of MegaSonic process conditions on PRE and sub-resolution assist feature damage [7122-35]

S. Helbig, HamaTech APE (Germany); S. Urban, E. Klein, Advanced Mask Technology Ctr. (Germany); S. Singh, Hamatech USA, Inc. (United States)

712211 Tunable droplet momentum and cavitation process for damage-free cleaning of challenging particles [7122-36]

R. Gouk, J. Papanu, F. Li, J. Jeon, T. Liu, R. Yalamanchili, Applied Materials (United States)

712212 Phase shift improvement in ArF/KrF haze-free mask cleaning [7122-37]

C. Liu, S. Zhao, E. Guo, Semiconductor Manufacturing International Corp. (China);

S. Hasegawa, K. Nemoto, T. Kuwajima, Tsukuba Semi Technology, Inc. (Japan)

\section{SESSION 9 HAZE CONTAMINATION CONTROL}

712213 Crystal growth printability in an advanced foundry FAB: a correlation study between STARlight and ultra broadband BrightField inspection technologies [7122-38]

T. H. Ng, M. F. bin Rahmat, Chartered Semiconductor Manufacturing, Ltd. (Singapore); B. Saville, P. Pak, W. Chia, A. Chin, M. VanRiet, R. Dover, R. Badoni, KLA-Tencor Corp. (United States)

712215 Report of latent contamination factors inducing lithographic variation [7122-40] J. H. Ryu, K. J. Seo, J. S. Ryu, C. Y. Kim, Hynix Semiconductor, Inc. (Korea, Republic of) 
712216 Detection of progressive transmission loss due to haze with Galileo mask DUV transmittance mapping based on non imaging optics [7122-41]

S. Labovitz, G. Ben-Zvi, V. Dmitriev, E. Graitzer, E. Zait, Pixer Technology (Israel)

712217 A new paradigm for haze improvement: retardation of haze occurrence by creating mask substrate insensitive to chemical contamination level [7122-42]

H. Lee, J. Choi, J. Jung, J. Oh, S. Kang, H. Jeong, S.-G. Woo, H. Cho, SAMSUNG Electronics Co., Ltd. (Korea, Republic of)

712218 Simulation analysis of backside defects printability in 193nm photolithography [7122-43] J. Ryu, D. Lee, J. Ryu, S. Jeong, S. Kim, C. Kim, Hynix Semiconductor, Inc. (Korea, Republic of)

712219 Optimal mask characterization by Surrogate Wafer Print (SWaP) method [7122-44] K. R. Kimmel, I. Hoellein, J. H. Peters, Advanced Mask Technology Ctr. GmbH \& Co. KG (Germany); P. Ackmann, Advanced Micro Devices, Inc. (United States); B. Connolly, C. West, Toppan Photomasks, Inc. (Germany)

$71221 \mathrm{~A}$ High resolution inspection with wafer plane die: database defect detection [7122-45] C. Hess, M. Wihl, R. Shi, Y. Xiong, S. Pang, KLA-Tencor Corp. (United States)

7122 1B Wafer plane inspection evaluated for photomask production (First Place Best Paper Award) [7122-46]

E. Gallagher, K. Badger, M. Lawliss, IBM Corp. (United States); Y. Kodera, Toppan Photomask, Inc. (United States); J. T. Azpiroz, IBM Corp. (United States); S. Pang, H. Zhang, E. Eugenieva, C. Clifford, A. Goonesekera, Y. Tian, KLA-Tencor Corp. (United States)

7122 1C Wafer plane inspection with soft resist thresholding [7122-47] C. Hess, R. Shi, M. Wihl, Y. Xiong, S. Pang, KLA-Tencor Corp. (United States)

7122 1D AIMS and resist simulation [7122-48] U. Strößner, H. Seitz, R. Birkner, R. Richter, T. Scherübl, Carl Zeiss SMS GmbH (Germany)

7122 1E AIMS-45 image validation of contact hole patterns after inverse lithography at NA 1.35 [7122-49]

E. HendrickX, IMEC (Belgium); R. Birkner, Mentor Graphics Corp. (United States);

M. Kempsell, A. Tritchkov, Carl Zeiss SMS (Germany); G. Vandenberghe, IMEC (Belgium); T. Schervebl, Mentor Graphics Corp. (United States)

\section{SESSION 11 INSPECTION}

$71221 \mathrm{~F}$ Auto-classification and simulation of mask defects using SEM and CAD images [7122-122] T.-Y. Kang, H.-C. Lee, Taiwan Semiconductor Manufacturing Co. Ltd. (Taiwan); H. Zhang, K. Yamada, Y. Kitayama, K. Kobayashi, Holon Co., Ltd. (Japan); P. Fiekowsky, Automated Visual Inspection LLC (United States) 
$71221 G \quad$ Novel mask inspection flow using Sensitivity Control Layers (SCL) on the TeraScanHR-587 platform [7122-51]

S. Hedges, Phontronics nanoFab North America (United States); C. Le, Micron Technology (United States); M. Eickhoff, M. Wylie, KLA-Tencor Corp. (United States); T. Simmons, Micron Technology (United States); V. Vellanki, KLA-Tencor Corp. (United States); J. McMurran, Phontronics nanoFab North America (United States)

$7122 \mathrm{1H} \quad$ Evaluating practical vs. theoretical inspection system capability with a new programmed defect test mask designed for $3 X$ and $4 X$ technology nodes [7122-52]

J. Glasser, T. Pratt, KLA-Tencor Corp. RAPID (United States)

\section{SESSION 12 DEFECT REPAIR}

$712211 \quad$ Nanomachining photomask repair of complex patterns [7122-53]

T. Robinson, A. Dinsdale, M. Archuletta, R. Bozak, R. White, RAVE LLC (United States)

$71221 \mathrm{~J}$ Practical laser mask repair in the contemporary production environment [7122-54] T. Robinson, R. White, M. Archuletta, R. Bozak, RAVE LLC (United States)

$71221 \mathrm{~K}$ Advanced process capabilities for electron beam based photomask repair in a production environment [7122-55]

A. Garetto, Carl Zeiss SMT, Inc. (United States); C. Baur, J. Oster, NaWoTec GmbH (Germany); M. Waiblinger, Carl Zeiss SMS GmbH (Germany); K. Edinger, NaWoTec GmbH (Germany)

\section{SESSION 13 RET AND OPC/ORC II}

7122 IL 32nm 1-D regular pitch SRAM bitcell design for interference-assisted lithography [7122-56] R. T. Greenway, Petersen Advanced Lithography (United States); K. Jeong, A. B. Kahng, C.-H. Park, Univ. of California, San Diego (United States); J. S. Petersen, Petersen Advanced Lithography (United States)

$71221 \mathrm{M}$ Convergence-based OPC method for dense simulations [7122-57]

T. Desouky, Mentor Graphics Egypt (Egypt)

$71221 \mathrm{~N}$ Combination of rule and pattern based lithography unfriendly pattern detection in OPC flow [7122-58]

J.-H. Kang, J.-Y. Choi, Y.-A. Shim, H.-S. Lee, Dongbu HiTek Co., Ltd. (South Korea); B. Su, W. Chan, P. Zhang, J. Wu, Anchor Semiconductor (United States); K.-Y. Kim, International Technology Alliances, Inc. (United States)

712210 Automated OPC model collection, cleaning, and calibration flow [7122-59] M. Drapeau, Synopsys, Inc. (Canada); B. S. Ward, Synopsys, Inc. (Belgium); B. Falch, Synopsys, Inc. (United States) 
$71221 Q \quad$ Improvements in accuracy of dense OPC models [7122-61]

C. Kallingal, Advanced Micro Devices, Inc. (United States); J. Oberschmidt, R. Viswanathan, A. Abdo, IBM (United States); O. Park, Infineon Technologies NA Corp. (United States)

$71221 R$ Defining a physically accurate laser bandwidth input for optical proximity correction (OPC) and modeling [7122-62]

I. Lalovic, Cymer, Inc. (United States); O. Kritsun, S. McGowan, Advanced Micro Devices (United States); J. Bendik, Dynamic Intelligence, Inc. (United States); M. Smith, KLA-Tencor (United States); N. Farrar, Cymer, Inc. (United States)

7122 is Benchmark of rigorous methods for electromagnetic field simulations [7122-63] S. Burger, L. Zschiedrich, F. Schmidt, Zuse Institute Berlin (Germany) and JCMwave GmbH (Germany); P. Evanschitzky, A. Erdmann, Fraunhofer Institute of Integrated Systems and Device Technology (Germany)

7122 1T Fast and accurate hybrid subgrid and subcell finite-difference time-domain methods for the simulation of mask electromagnetic effects in sub-45nm lithography [7122-64] M. S. Yeung, Fastlitho, Inc. (United States)

$71221 \mathrm{U}$ An efficient method for transfer cross coefficient approximation in model based optical proximity correction [7122-142]

R. Sabatier, Institut Fresnel, École Centrale Marseille, CNRS (France) and STM (France);

C. Fossati, S. Bourennane, Institut Fresnel, École Centrale Marseille, CNRS (France);

A. Di Giacomo, STM (France)

\section{SESSION 15 SIMULATION AND MODELING II}

$71221 \mathrm{~V}$ Scanner-specific separable models for computational lithography [7122-66]

S. Hunsche, X. Xie, Q. Zhao, H.-Y. Liu, Brion Technologies (United States); P. Nikolsky, A. Ngai, ASML Netherlands B.V. (Netherlands)

7122 IW Considering MEEF in inverse lithography technology (ILT) and source mask optimization (SMO) [7122-68]

L. Pang, G. Xiao, V. Tolani, P. Hu, T. Cecil, T. Dam, K.-H. Baik, B. Gleason, Luminescent Technologies, Inc. (United States)

$71221 \mathrm{X}$ Smoothing based model for images of buried EUV multilayer defects near absorber features [7122-69]

C. H. Clifford, A. R. Neureuther, Univ. of California, Berkeley (United States)

7122 1Y Simulation-based EUV source and mask optimization [7122-70]

T. Fühner, A. Erdmann, P. Evanschitzky, Fraunhofer Institute of Integrated Systems and Device Technology (Germany) 
712212 DFM viewpoints of cell-level layout assessments and indications for concurrent layout optimization [7122-71]

C.-M. Fu, P.-H. Yeh, Y.-K. Cheng, Taiwan Semiconductor Manufacturing Co., Ltd. (Taiwan);

S. Klaver, Takumi Technology Corp. (United States)

712220 Concurrent optimization of MDP, mask writing, and mask inspection for mask manufacturing cost reduction [7122-72]

M. Yamabe, T. Inove, M. Shoji, H. Yasuda, H. Hoshi, M. Tokita, Association of SuperAdvanced Electronics Technologies (Japan)

712221 Design for manufacturability guideline development: integrated foundry approach [7122-73]

H. Lee, Y.-A. Shim, J.-Y. Choi, K.-S. Choi, Dongbu HiTek (Korea, Republic of); J. Wu, B. Su, $X$. Zhou, Anchor Semiconductor, Inc. (United States); K. Kim, International Technology Alliances, Inc. (United States)

\section{Part Two}

712222 Flexible sensitivity inspection with TK-CMI software for criticality-awareness [7122-74] F. A. J. M. Driessen, J. Gunawerdana, Takumi Technology Corp. (United States); Y. Saito, H. Tsuchiya, NuFlare Technology, Inc. (Japan); Y. Tsuji, Advanced Mask Inspection Technology, Inc. (Japan)

712223 Improving contact and via process latitude through selective upsizing [7122-75] C. Yuan, G. Abeln, B. Anthony, G. Chen, S. Robertson, P. Walker, Freescale Semiconductor (United States)

712224 Assist feature aware double patterning decomposition [7122-76]

C. Cork, Synopsys SARL (France); L. Barnes, G. Luk-Pat, Synopsys, Inc. (United States)

\section{SESSION 17 EUV MASK PROCESSING AND SUBSTRATES}

712225 Development status of EUVL mask blanks [7122-77]

K. Shiromo, Asahi Glass Co., Ltd. (Japan)

712226 Chemical durability studies of Ru-capped EUV mask blanks [7122-78]

T. Shimomura, Dai Nippon Printing Co., Ltd. (Japan); T. Liang, Intel Corp. (United States)

712227 EUVL practical mask structure with light shield area for $32 \mathrm{~nm}$ half pitch and beyond [7122-79]

T. Kamo, H. Aoyama, T. Tanaka, O. Suga, MIRAI-Semiconductor Leading Edge Technologies, Inc. (Japan); T. Abe, T. Takikawa, N. Hayashi, Dai Nippon Printing Co., Ltd. (Japan); T. Shoki, Y. Usui, M. Hosoya, HOYA Corp. (Japan) 
712228 Control of the sidewall angle of an absorber stack using the Faraday cage system for the change of pattern printability in EUVL [7122-80]

I.-Y. Jang, S.-M. Huh, S.-Y. Moon, S.-G. Woo, SAMSUNG Electronics Co., Ltd. (Korea, Republic of); J.-K. Lee, S. H. Moon, Seoul National Univ. (Korea, Republic of); H. Cho, SAMSUNG Electronics Co., Ltd. (Korea, Republic of)

\section{SESSION 18 EUV MASK PROCESS CORRECTION}

7122 2C Pattern placement correction due to bending in EUVL masks [7122-84]

S.-J. Park, C. Manish, M. Kamna, C. Hu, G. Zhang, F. C. Martinez, N. Wilcox, K. Hsia,

A. R. Stivers, Intel Corp. (United States)

\section{SESSION 19 EUV MASK INSPECTION I}

7122 2D Signal analysis for the actinic full-field EUVL mask blank inspection system [7122-85] T. Yamane, T. Iwasaki, T. Tanaka, T. Terasawa, O. Suga, MIRAI-Semiconductor Leading Edge Technologies, Inc. (Japan); T. Tomie, National Institute of Advanced Industrial Science and Technology (Japan)

7122 2E Benchmarking EUV mask inspection beyond 0.25 NA [7122-86]

K. A. Goldberg, I. Mochi, P. P. Naulleau, Lawrence Berkeley National Lab. (United States);

H. Han, SAMSUNG Electronics Co., Ltd. (Korea, Republic of); S. Huh, SEMATECH (United States)

\section{SESSION 20 EUV MASK INSPECTION II}

$71222 \mathrm{~F}$ The study of EUVL mask defect inspection technology for 32-nm half-pitch node device and beyond [7122-87]

H. Shigemura, T. Amano, Y. Nishiyama, O. Suga, T. Terasawa, Y. Arisawa, MIRAI-Semiconductor Leading Edge Technologies, Inc. (Japan); H. Hashimoto, N. Kameya, M. Takeda, NuFlare Technology, Inc. (Japan); N. Kikuiri, R. Hirano, M. Hirono, Advanced Mask Inspection Technology, Inc. (Japan)

$71222 \mathrm{G} \quad$ An investigation of EUV lithography defectivity [7122-88]

K. D. Cummings, T. Laursen, B. Pierson, S. Han, R. Watso, Y. van Dommelen, B. Lee, ASML (United States); Y. Deng, B. La Fontaine, T. Wallow, U. Okoroanyanwu, O. Wood, Advanced Micro Devices (United States); A. Tchikoulaeva, AMD Fab36 LLC (Germany); C. Holfeld, J. H. Peters, Advanced Mask Technology Ctr. (Germany); C. Koay, K. Petrillo, IBM Corp. (United States); T. DiBiase, S. Kini, KLA-Tencor Corp. (United States); H. Mizuno, Toshiba America Electronics Components (United States)

\section{SESSION 21 EUV MASK REPAIR}

$71222 \mathrm{H} \quad$ Study of EUVL mask defect repair using FIB-GAE method [7122-89]

T. Amano, Y. Nishiyama, H. Shigemura, T. Terasawa, O. Suga, MIRAI-Semiconductor Leading Edge Technologies, Inc. (Japan); K. Shiina, F. Aramaki, R. Hagiwara, A. Yasaka, SII NanoTechnology, Inc. (Japan) 
$712221 \quad$ Analysis of process margin in EUV mask repair with nano-machining [7122-90] S.-Y. Lee, G.-B. Kim, H.-S. Sim, S.-H. Lee, H.-S. Kim, J.-H. Lee, H.-S. Seo, H.-S. Han, S.-S. Kim, S.-Y. Moon, S.-G. Woo, SAMSUNG Electronics Co., Ltd. (Korea, Republic of); R. Bozak, A. Dinsdale, T. Robinson, D. Lee, RAVE LLC (United States); H. Cho, SAMSUNG Electronics Co., Ltd. (Korea, Republic of)

7122 2J Ga implantation and interlayer mixing during FIB repair of EUV mask defects [7122-91] Y. Nishiyama, T. Amano, H. Shigemura, T. Terasawa, O. Suga, MIRAI-Semiconductor Leading Edge Technologies, Inc. (Japan); T. Kozakai, F. Aramaki, K. Shiina, A. Yasaka, R. Hagiwara, SIl NanoTechnology, Inc. (Japan)

\section{SESSION 22 MASKS FOR NANO-IMPRINT LITHOGRAPHY}

7122 2K Defect inspection of imprinted $32 \mathrm{~nm}$ half pitch patterns [7122-92]

K. Selinidis, E. Thompson, I. McMackin, J. Perez, S. V. Sreenivasan, D. J. Resnick, Molecular Imprints, Inc. (United States)

$71222 \mathrm{~L} \quad$ Evaluation of e-beam repair for nanoimprint templates [7122-93]

M. Pritschow, IMS Chips (Germany); V. Boegli, NaWoTec GmbH (Germany); J. Butschke, M. Irmscher, IMS Chips (Germany); D. Resnick, Molecular Imprints, Inc. (United States); H. Sailer, IMS Chips (Germany); K. Selinidis, E. Thompson, Molecular Imprints, Inc. (United States)

\section{SESSION 23 MASK BUSINESS}

7122 2M Diamonds in the rough: key performance indicators for reticles and design sets [7122-94] P. Ackmann, Advanced Micro Devices, Inc. (United States)

7122 2N Accelerated data communication of semiconductor design files [7122-95] G. H. Fairbank, Saratoga Data Systems, Inc. (United States)

712220 Compute resource management and turn around time control in mask data prep [7122-96] T. Lewis, S. Goad, AMD (United States); K. Jantzen, A. Nouh, M. Park, E. Sahouria, S. Schulze, Mentor Graphics Corp. (United States)

\section{SESSION 24 SEM CD METROLOGY}

7122 2P International photomask linewidth comparison by NIST and PTB [7122-97]

J. Potzick, R. Dixson, R. Quintanilha, M. Stocker, A. Vladar, National Institute of Standards and Technology (United States); E. Buhr, W. Häßler-Grohne, B. Bodermann, C. G. Frase, H. Bosse, Physikalisch-Technische Bundesanstalt (Germany)

$71222 \mathrm{R}$ Re-calibration of the NIST SRM 2059 master standard using traceable atomic force microscope metrology [7122-98]

R. Dixson, J. Potzick, N. G. Orji, National Institute of Standards and Technology (United States) 
$71222 R \quad$ A study of the limited area scanning system in the mask CD-SEM [7122-99]

T. Iwai, S. Shida, M. Hiroyama, T. Nakamura, Advantest Corp. (Japan); H. Sakaguchi, H. Ueno, Vistec Semiconductor Systems K.K. (Japan); M. Higuchi, T. Aihara, Toppan Printing Co., Ltd. (Japan)

$712225 \quad$ Exploring new metrology for complex photomask patterns [7122-100]

M. Higuchi, T. Aihara, H. Saito, I. Yonekura, J. Suzuki, Toppan Printing Co., Ltd. (Japan);

E. Gallagher, I. P. Stobert, IBM Corp. (United States)

7122 2T New method of 2-dimensional metrology using mask contouring [7122-101]

R. Matsuoka, Y. Yamagata, A. Sugiyama, Hitachi High-Technologies Corp. (Japan);

Y. Toyoda, Hitachi, Ltd. (Japan)

$71222 \mathrm{U}$ Comparative scatterometric CD measurements on a MoSi photo mask using different metrology tools [7122-102]

J. Richter, J. Rudolf, Advanced Mask Technology Ctr. GmbH \& Co. KG (Germany);

B. Bodermann, Physikalisch-Technische Bundesanstalt (Germany); J. C. Lam, n\&k

Technology, Inc. (United States)

7122 2V Mask process monitoring with optical CD measurements for sub-50-nm [7122-103] K.-Y. Bang, J.-B. Park, J.-H. Roh, D.-H. Chung, S.-Y. Cho, Y.-H. Kim, S.-G. Woo, H.-K. Cho, SAMSUNG Electronics Co., Ltd. (Korea, Republic of)

7122 2X Improvement in metrology on new 3D-AFM platform [7122-105]

I. Schmitz, M. Osborn, S. Hand, Q. Chen, Veeco Metrology (United States)

\section{SESSION 26 METROLOGY FOR PLACEMENT AND OPTICAL STRUCTURE}

7122 2Y Development of a 1.5D reference comparator for position and straightness metrology on photomasks [7122-106]

J. Flügge, R. Köning, Ch. Weichert, W. Häßler-Grohne, R. D. Geckeler, A. Wiegmann, M. Schulz, C. Elster, H. Bosse, Physikalisch-Technische Bundesanstalt (Germany)

712222 Photomask registration and overlay metrology by means of $193 \mathrm{~nm}$ optics [7122-107] G. Klose, N. Kerwien, M. Arnz, Carl Zeiss SMT AG (Germany); D. Beyer, N. Rosenkranz, Carl Zeiss SMS GmbH (Germany)

712230 World wide matching of registration metrology tools of various generations [7122-108] F. Laske, Vistec Semiconductor Systems GmbH (Germany); A. Pudnos, L. Mackey, P. Tran, Toppan Photomasks, Inc. (United States); M. Higuchi, Toppan Printing Co., Ltd. (Japan); C. Enkrich, Advanced Mask Technology Ctr. Dresden (Germany); K.-D. Roeth, K.-H. Schmidt, D. Adam, J. Bender, Vistec Semiconductor Systems GmbH (Germany)

712231 Spectroscopic ellipsometry applications in photomask technology [7122-109]

R. A. Synowicki, J. N. Hilfiker, J.A. Woollam Co., Inc. (United States) 
712233 True reticle cost saving by multi level reticle approach [7122-111]

T. Struck, Infineon Technologies AG (Germany); H. Kirbach, Infineon Technologies Dresden $\mathrm{GmbH} \& \mathrm{Co}$. OHG (Germany)

712234 Technology interactions on reticle delivery [7122-112]

P. Ackmann, S. Goad, Advanced Micro Devices, Inc. (United States); C. West, Toppan Photomasks (United States)

\section{POSTER SESSION: ADVANCED MASK PATTERNING}

712235 Deflection unit for multi-beam mask making (First Place Best Poster Award) [7122-1 14]

F. Letzkus, J. Butschke, M. Irmscher, M. Jurisch, W. Klingler, IMS Chips (Germany);

E. Platzgummer, C. Klein, H. Loeschner, R. Springer, IMS Nanofabrication AG (Austria)

712236 Mask patterning for the $22 \mathrm{~nm}$ node using a proton multi-beam projection pattern generator (Second Place Best Poster Award) [7122-115]

J. Butschke, M. Irmscher, H. Sailer, L. Nedelmann, M. Pritschow, IMS Chips (Germany);

H. Loeschner, E. Platzgummer, IMS Nanofabrication AG (Austria)

712237 High accuracy jog CD control on OPC pattern by advanced laser writer Sigma7500 [7122-116]

T. Chin, W.-B. Wu, C.-L. Shih, Nanya Technology Corp. (Taiwan)

POSTER SESSION: ADVANCED MASK PROCESSING AND MATERIALS

712238 70nm DRAM intra-field CDU improvement by dose modulation on mask transmittance [7122-117]

T. Chin, W. B. Wu, C. L. Shih, P. C. Fan, Nanya Technology Corp. (Taiwan); G. B. Zvi, Pixer

Technology, Ltd. (Israel)

712239 The study of CD behavior due to transmission control position change within photomask substrate [7122-118]

M. Kim, H. Lee, S. Woo, K. Seo, Y. Choi, C. Kim, Hynix Semiconductor, Inc. (South Korea)

$71223 \mathrm{~A}$ Effects of photo resist erosion in development on critical dimension performance for $45 \mathrm{~nm}$ node and below [7122-119]

G.-H. Hwang, D.-H. Kim, C. YU, B.-S. Kang, I.-B. Hur, C. Shin, S.-M. Jung, S.-S. Choi, PKL (Korea, Republic of)

\section{POSTER SESSION: METROLOGY}

$71223 \mathrm{C}$ Novel CD measurement and precise pattern size extraction method for optical images [7122-104]

L. Faivishevsky, Applied Materials (Israel) 
$71223 \mathrm{E} \quad$ A novel approach to mask defect inspection [7122-50]

A. Sagiv, Y. Shirman, S. Mangan, Applied Materials (Israel)

$71223 \mathrm{~F} \quad$ Automated reticle inspection data analysis for wafer fabs [7122-124]

D. Summers, G. Chen, Freescale-ATMC (United States); B. Reese, T. Hutchinson,

M. Liesching, H. Ying, R. Dover, KLA-Tencor Corp. (United States)

$71223 G \quad$ Results from the KLA-Tencor TeraScanXR reticle inspection tool [7122-125]

A. Dayal, B. Mu, V. Iyer, P. Lim, A. Goonesekera, B. Broadbent, KLA-Tencor Corp. (United States)

$71223 \mathrm{H} \quad$ Layout driven DNIR [7122-126]

K. Kageyama, K. Yanagisawa, A. Kobayashi, S. Kunitani, Y. Tonooka, Toppan Printing Co., Ltd. (Japan)

7122 3J Improving cost of ownership on KLA-Tencor wafer fab reticle inspections by implementing pixel migration via new STARlight2+ capability [7122-128]

Y.-F. Cheng, W.-C. LO, M.-J. Chen, P. Huang, United Microelectronics Corp. (Taiwan);

C. Chen, S. Chakravarty, P. Yu, R. Dover, KLA-Tencor Corp. (United States)

\section{POSTER SESSION: CONTAMINATION CONTROL AND CLEANING}

$71223 \mathrm{~L} \quad$ An effective haze monitoring method [7122-130]

S.-P. Lu, S.-H. Chiou, W.-J. Tseng, Rexchip Electronic Corp. (Taiwan)

$71223 \mathrm{M} \quad$ Results of new mask contamination inspection capability STARlight2+ $72 \mathrm{~nm}$ pixel with cell-to-cell HiRes5 for qualifying memory masks in wafer fabs [7122-131]

R. Badoni, J. Zhu, R. Dover, N. Schmidt, M. Lang, KLA-Tencor Corp. (United States);

A. Jahnke, F. Uhlig, Qimonda Dresden GmbH \& Co. OHG (United States)

7122 3N SL2+: H5 use case [7122-132]

K. Ito, S. Liu, I. Lee, R. Dover, P. Yu, KLA-Tencor Corp. (United States)

\section{POSTER SESSION: EUV MASK PROCESSING AND SUBSTRATES}

712230 Evaluation of backside particle contamination and electrostatic chuck design on the cleanliness of EUV reticle mask blanks in a multilayer Mo/Si ion beam deposition system [7122-133]

A. V. Hayes, R. Randive, I. Reiss, J. Menendez, Veeco Instruments (United States);

P. Kearney, T. Sugiyama, SEMATECH (United States)

\section{POSTER SESSION: NANO-IMPRINT}

$71223 \mathrm{P}$ UV NIL template making and imprint evaluation (Third Place Best Poster Award) [7122-135]

S. Sasaki, T. Hiraka, J. Mizuochi, A. Fujii, Y. Sakai, T. Sutou, S. Yusa, K. Kuriyama, M. Sakaki,

Y. Morikawa, H. Mohri, N. Hayashi, Dai Nippon Printing Co., Ltd. (Japan) 
$71223 Q \quad$ Development status of back-end process for UV-NIL template fabrication [7122-136]

Y. Inazuki, K. Itoh, S. Hatakeyama, K. Kojima, M. Kurihara, Y. Morikawa, H. Mohri, N. Hayashi,

Dai Nippon Printing Co., Ltd. (Japan)

\section{POSTER SESSION: DFM}

$71223 R \quad$ Non-uniform yield optimization for integrated circuit layout considering global interactions [7122-137]

J. A. Torres, F. G. Pikus, Mentor Graphics Corp. (United States)

$712235 \quad$ Fast and simple modeling of non-rectangular transistors [7122-138]

J.-Y. WuU, Univ. of California, Santa Barbara (United States); F. G. Pikus, Mentor Graphics Corp. (United States); M. Marek-Sadowska, Univ. of California, Santa Barbara (United States)

7122 3T Study of influence to transistor properties on the change of OPC pattern [7122-139]

K. Sugawa, N. Nagase, T. Itoh, M. Sakurai, T. Okada, Fujitsu Microelectronics, Ltd. (Japan)

$71223 \mathrm{U}$ Mask data prioritization based on design intent [7122-140]

K. Kato, M. Endo, T. Inove, M. Yamabe, Association of Super-Advanced Electronics

Technologies (Japan)

POSTER SESSION: SIMULATION AND MODELING

7122 3W Extracting mask error function from intensity slices [7122-65]

D. Ziger, Synopsys, Inc. (United States)

$71223 Y \quad$ Focus blur model to enhance lithography model for optical proximity correction [7122-144] Q. Zhang, H. Song, K. Lucas, Synopsys, Inc. (United States)

POSTER SESSION: RET AND OPC/ORC

$712232 \quad 32 \mathrm{~nm}$ design rule and process exploration flow [7122-33]

Y. Zhang, J. Cobb, A. Yang, J. Li, K. Lucas, S. Sethi, Synopsys, Inc. (United States)

712240 Accelerate OPC convergence with new iteration control methodology [7122-145] C.-H. Wang, Q. Liu, Semiconductor Manufacturing International Corp. (China); L. Zhang, Mentor Graphics Corp. (China)

712241 Enhanced DCT2-based inverse mask synthesis with initial SRAF insertion [7122-146] S. Shen, Zhejiang Univ. (China); P. YU, D. Z. Pan, The Univ. of Texas at Austin (United States)

$712242 \quad$ Empirical study of OPC metrology requirements for 32-nm node logic [7122-147] B. S. Ward, IMEC (Belgium); L. Zavylova, Synopsys (United States); P. de Bisschop, J. van de Kerkhove, IMEC (Belgium)

712243 Adaptive automatic fragmentation [7122-148]

M. S. E.-D. Habib, Mentor Graphics Corp. (United States) 
712244 Enhancing OPC model stability and predictability using SEM image contours [7122-149] M. S. E.-D. Habib, Mentor Graphics Corp. (United States)

712245 Characterizing OPC model accuracy versus lens induced polarization effects in hyper NA immersion lithography [7122-150]

T. M. Tawfik, E. Tejnil, Mentor Graphics Corp. (United States)

712248 Toward faster OPC convergence: advanced analysis for OPC iterations and simulation environment [7122-154]

M. Bahnas, M. Al-Imam, T. Tawfik, Mentor Graphics Corp. (Egypt)

712249 OPC cycle time reduction and accuracy improvement by early access to advanced Tachyon modeling of TWINSCAN XT:1900i scanner [7122-155]

P. Nikolsky, T. Nooitgedagt, P. van Adrichem, J. Meessen, C. Kohler, B. Wisse, ASML Netherlands B.V. (Netherlands); S. Hunsche, Brion (United States)

7122 4A Sizing algorithm with continuous customizable clipping [7122-157]

D. Morales, F. Baytelman, H. Araya, Synopsys Chile Research and Development Ctr. (Chile)

Author Index 
Downloaded From: https://www.spiedigitallibrary.org/conference-proceedings-of-spie on 26 Apr 2023

Terms of Use: https://www.spiedigitallibrary.org/terms-of-use 


\title{
Conference Committee
}

\author{
Conference Chair
}

Hiroichi Kawahira, Sony Corporation (Japan)

Conference Cochair

Larry S. Zurbrick, Agilent Technologies, Inc. (United States)

Program Committee

Frank Abboud, Intel Corporation (United States)

Kiho Baik, Luminescent Technologies, Inc. (United States)

Artur P. Balasinski, Cypress Semiconductor Corporation (United States)

Uwe F. W. Behringer, UBC Microelectronics (Germany)

Ronald R. Bozak, RAVE LLC (United States)

William H. Broadbent, KLA-Tencor Corporation (United States)

Peter D. Buck, Toppan Photomasks, Inc. (United States)

Han-Ku Cho, SAMSUNG Electronics Company, Ltd. (Korea, Republic of)

Frank A. Driessen, Takumi Technology B.V. (Netherlands)

Roxann L. Engelstad, University of Wisconsin, Madison (United States)

Donis G. Flagello, ASML US, Inc. (United States)

Emily E. Gallagher, IBM Corporation (United States)

Bernd Geh, Carl Zeiss SMT AG/ASML-TDC (United States)

Brian J. Grenon, Grenon Consulting, Inc. (United States)

Naoya Hayashi, Dai Nippon Printing Company, Ltd. (Japan)

Judith A. Huckabay, Cadence Design Systems, Inc. (United States)

Mark T. Jee, Hoya Corporation USA (United States)

Bryan S. Kasprowicz, Photronics, Inc. (United States)

Kurt R. Kimmel, Advanced Mask Technology Center GmbH \& Company KG (Germany)

Chin-Hsiang Lin, Taiwan Semiconductor Manufacturing Company, Ltd. (Taiwan)

Mark E. Mason, Texas Instruments, Inc. (United States)

Wilhelm Maurer, Infineon Technologies AG (Germany)

M. Warren Montgomery, CNSE/SEMATECH (United States)

Thomas H. Newman, Micronic Laser Systems, Inc. (United States)

Hiroshi Nozue, NuFlare Technology, Inc. (Japan)

Emmanuel Rausa, Oerlikon USA, Inc. (United States)

Frank M. Schellenberg, Mentor Graphics Corporation (United States)

Steven D. Slonaker, Nikon Precision, Inc. (United States)

Christopher A. Spence, Advanced Micro Devices, Inc. (United States) 
Wolfgang Staud, B2W Consulting (United States)

Geert Vandenberghe, IMEC (Belgium)

J. Tracy Weed, Synopsys, Inc. (United States)

John M. Whittey, Vistec Semiconductor Systems, Inc. (United States)

\author{
Session Chairs \\ 1 Invited Session \\ Hiroichi Kawahira, Sony Corporation (Japan) \\ Larry S. Zurbrick, Agilent Technologies, Inc. (United States) \\ 2 Mask Materials for Optical Extensions \\ Emily E. Gallagher, IBM Corporation (United States) \\ Mark T. Jee, Hoya Corporation USA (United States) \\ 3 Advanced Mask Processing \\ Craig A. West, Toppan Photomasks, Inc. (United States) \\ Emmanuel Rausa, Oerlikon USA, Inc. (United States) \\ $4 \quad$ Patterning Technologies and Tools \\ Thomas H. Newman, Micronic Laser Systems, Inc. (United States) \\ Hiroshi Nozue, NuFlare Technology, Inc. (Japan) \\ 5 DPL Implementation I \\ Donis G. Flagello, ASML US, Inc. (United States) \\ Jacek K. Tyminski, Nikon Precision, Inc. (United States) \\ 6 DPL Implementation II \\ Donis G. Flagello, ASML US, Inc. (United States) \\ Jacek K. Tyminski, Nikon Precision, Inc. (United States) \\ $7 \quad$ RET and OPC/ORC I \\ Wilhelm Maurer, Infineon Technologies AG (Germany) \\ Christopher A. Spence, Advanced Micro Devices, Inc. (United States) \\ $8 \quad$ Advanced Cleaning \\ M. Warren Montgomery, CNSE/SEMATECH (United States) \\ Craig A. West, Toppan Photomasks, Inc. (United States) \\ 9 Haze Contamination Control \\ Brian J. Grenon, Grenon Consulting, Inc. (United States) \\ Naoya Hayashi, Dai Nippon Printing Company, Ltd. (Japan) \\ 10 Wafer Plane Inspection \\ William H. Broadbent, KLA-Tencor Corporation (United States) \\ Frank M. Schellenberg, Mentor Graphics Corporation (United States)
}


Inspection

William H. Broadbent, KLA-Tencor Corporation (United States)

John M. Whittey, Vistec Semiconductor Systems, Inc. (United States)

Defect Repair

Uwe F. W. Behringer, UBC Microelectronics (Germany)

Ronald R. Bozak, RAVE LLC (United States)

13 RET and OPC/ORC II

Frank M. Schellenberg, Mentor Graphics Corporation (United States)

Chan-Uk Jeon, SAMSUNG Electronics Company, Ltd. (Korea, Republic of)

14 Simulation and Modeling I

Christopher A. Spence, Advanced Micro Devices, Inc. (United States)

Bernd Geh, ASML US, Inc. (United States)

15 Simulation and Modeling II

J. Tracy Weed, Synopsys, Inc. (United States)

Kiho Baik, Luminescent Technologies, Inc. (United States)

16 DFM

Judith A. Huckabay, Cadence Design Systems, Inc. (United States)

Frank A. Driessen, Takumi Technology B.V. (Netherlands)

17 EUV Mask Processing and Substrates

Kurt R. Kimmel, Advanced Mask Technology Center GmbH \& Company KG (Germany)

18 EUV Mask Process Correction

Naoya Hayashi, Dai Nippon Printing Co., Ltd. (Japan)

19 EUV Mask Inspection I

Kurt R. Kimmel, Advanced Mask Technology Center GmbH \& Company KG (Germany)

M. Warren Montgomery, CNSE/SEMATECH (United States)

20 EUV Mask Inspection II

Kurt R. Kimmel, Advanced Mask Technology Center GmbH \& Company KG (Germany)

M. Warren Montgomery, CNSE/SEMATECH (United States)

21 EUV Mask Repair

Uwe F. W. Behringer, UBC Microelectronics (Germany)

Ronald R. Bozak, RAVE LLC (United States) 
22 Masks for Nano-Imprint Lithography

Larry S. Zurbrick, Agilent Technologies, Inc. (United States)

Bryan S. Kasprowicz, Photronics, Inc. (United States)

23 Mask Business

Larry S. Zurbrick, Agilent Technologies, Inc. (United States)

Bryan S. Kasprowicz, Photronics, Inc. (United States)

24 SEM CD Metrology

Frank Abboud, Intel Corporation (United States)

Emily E. Gallagher, IBM Corporation (United States)

25 Advanced CD Metrology

Thomas H. Newman, Micronic Laser Systems Inc. (United States)

Chan-Uk Jeon, SAMSUNG Electronics Company, Ltd. (Korea, Republic of)

26 Metrology for Placement and Optical Structure

John M. Whittey, Vistec Semiconductor Systems, Inc. (United States)

Wen-Hong Huang, Taiwan Semiconductor Manufacturing Company, Ltd. (Taiwan) 


\section{Introduction}

This proceedings volume contains accepted papers from the SPIE conference on Photomask Technology. The conference was arranged through the Bay Area Chrome Users Society (BACUS) and held as part of the 28th International Symposium on Photomask Technology 7-10 October 2008 in Monterey, California, USA.

The papers cover the most up-to-date research on emerging and ongoing issues facing the photomask industry in advanced lithography and their manufacturing solutions. With the deep sub-wavelength era upon us, the industry's progress will depend on the successful integration associated with optimization of design, mask making, and wafer fabrication. With respect to mask manufacturing infrastructure, topics are on advanced component processes, materials and patterning technologies for $32 \mathrm{~nm}$ node and beyond. In addition to them, special components for optical extension technologies, DFM (Design For Manufacturing) technologies being coupled with EDA (Electronic Design Automation) are also captured. More advanced technologies for realizing emerging technologies such as EUVL (Extreme Ultraviolet Lithography) and nanoimprint lithography are also covered. Lastly business aspects that examine cost friendly and manufacturable solutions for our industry are reviewed as well.

This year's symposium continued the fine tradition of having wide international representation with a number of papers specifically as following;

- Mask Infrastructure

- Mask Materials for Optical Extensions

- Advanced Mask Processing and Materials

- Patterning Technologies and Tools

- Metrology

- Defect Inspection

- Defect Repair

- Advanced Cleaning

- Haze Contamination Control

- Mask Integrations

- DPL (Double Patterning Lithography) Implementation

- DFM

- Simulation and Modeling

- RET (Resolution Enhancement Technology) and OPC (Optical Proximity Effect Correction)/ORC (Optical Rule Check)

- Wafer Plane Inspection

- Emerging Mask Technology

- EUV Mask Processing and Substrates

- EUV Mask Process Correction 


\section{- EUV Mask Inspection \\ - EUV Mask Repair \\ - Masks for Nano Imprint}

- Mask Business

- Industry Assessment

- Cost and Performance

- Data Process Management

This year's Special Session was on the topic "Mask Infrastructure Challenges at 32 $\mathrm{nm}$ and Beyond," where industry experts from around the world spanning DFM, mask making (captive and merchant), equipments, wafer manufacturing, technology consultant, and national research institute met to explore the current most up-to-dated status and critical issues for the future. The summary of this session is provided by Griff Resor this year.

I thank all of the authors, particularly the keynote speaker Dr. Aart de Geus, Chairman and CEO of Synopsys, Inc. who gave us a talk with deep insight on this industry and prospect for the future which was so much instructive and stimulating. I also thank all the members of program committee for their dedication and hard work to help maintain the high quality of this conference with chairing respective technical sessions. Among this, I am especially grateful to my cochair Larry S. Zurbrick, Agilent Technologies, Inc. for all his help in making this year's symposium a great success. Of course, the sponsors who allow us to continue to attract talented speakers deserve recognition for without them, we would not be able to exist as a conference. Finally, I extend my sincere appreciation to the SPIE staff for their extensive efforts and their superb organizational skills that helped make this year's SPIE Photomask Technology conference a success, including a special thank you to SPIE for assembling and publishing this proceedings volume.

I hope you find the material comprehensive and valuable to your technical field, whatever that may be. BACUS is a professional society. If you are interested in what we are about, please visit the technical group on the SPIE website at www.SPIE.org/BACUSHome and join us as we continue to influence photomask technology development through our respected career applications.

Hiroichi Kawahira 
Friday Special Session Schedule

\section{Mask Infrastructure Challenges at $32 \mathrm{~nm}$ and Beyond *}

Chairs: Larry S. Zurbrick, Agilent Technologies, Inc. (United States); Douglas J. Resnick, Molecular Imprints, Inc. (United States); Benjamin G. Eynon, Jr., Molecular Imprints, Inc. (United States); M. Warren Montgomery, CNSE/SEMATECH (United States)

Opening Remarks and Introduction

Larry S. Zurbrick, Agilent Technologies, Inc. (United States)

\section{Mask Technology Perspectives}

Software: Double Patterning and EUV Shadowing

Luigi Capodieci, Advanced Micro Devices, Inc. (United States)

E-beam PG: Resist, CD, CDU, IP, LWR

Shusuke Yoshitake, NuFlare Technology (Japan)

Pattern Transfer: CD bias, CDU, EPD

Thomas B. Faure, IBM Corp. (United States)

Inspection: E-beam versus Optical

Franklin D. Kalk, Toppan Photomasks, Inc. (United States)

Repair: Subtractive and Additive

Ted Liang, Intel Corp. (United States)

Metrology: CD, IP

Richard M. Silver, National Institute of Standards and Technology (United States)

Clean: Haze Issues etc.

Brian J. Grenon, Grenon Consulting, Inc. (United States)

\section{Lithography Perspectives}

Optical Extensions: Double Patterning

Paul W. Ackmann, Advanced Micro Devices, Inc. (United States)

EUV: Blank, Defectivity, PT

Henry Yun, SEMATECH (United States)

Imprint: Write, PT, Inspection, Repair

Naoya Hayashi, Dai Nippon Printing Co., Ltd. (Japan)

Open Discussion

* For materials relating to this session point your web browser to: $\underline{\text { wwW.SPIE.org/PM08 }}$
* For information about the BACUS technical group go to: www.SPIE.org/BACUSHome 
Downloaded From: https://www.spiedigitallibrary.org/conference-proceedings-of-spie on 26 Apr 2023

Terms of Use: https://www.spiedigitallibrary.org/terms-of-use 
Summary of the Friday Special Session

\section{To 32nm and beyond: SPIE panel debates assortment of challenges *}

What challenges must maskmakers overcome to reach the $32 \mathrm{~nm}$ node, and what challenges lie beyond? The BACUS Program Committee assembled a panel of 10 experts to answer these questions. This glance at our future brought many people to this year's SPIE Photomask Technology Conference (Oct 6-10, Monterey, CA).

Luigi Capodiece of AMD launched the discussion with a lively review of EDA challenges. At the $32 \mathrm{~nm}$ node, critical levels will be done with double or triple patterning. Manual methods are needed to decide which patterns go on each mask level. The design software then adds reticle enhancement (RET) detail based on tool and process models. The RET detail, sub-resolution assist features (SRAF) for example, can interact between mask levels. So careful simulation of this interaction is needed to avoid printing unwanted detail. More work by EDA suppliers is needed to make this work automatically; automatic routing is the most urgently needed capability.

Susuki Yoshitake of NuFlare described the company's work to develop adequate e-beam tools for mask writing. NuFlare clearly is supporting a large R\&D effort; its most recent model EB $6000+$ now meets $45 \mathrm{~nm}$ node requirements, the model $E B$ 7000 will be out next year (CY2009) for writing 32nm-node masks, and a model EB 8000 is planned for CY2011 for writing 22nm-node masks. As the data file size increases and the number of individual shaped spots increases; NuFlare expects to keep write time under 24 hours/mask. There are problems, though -- the company has been able to reduce the beam blur to less than 10nm, but current chemically amplified resist (CAR) then adds about $28 \mathrm{~nm}$ of "process blur." So, an improved CAR with less diffusion blur and less line-edge roughness (LER) is needed.

The problem of mask etching -- transferring the e-beam resist pattern into the chrome mask -- was highlighted by Tom Faure of IBM. Each new node requires thinner resist, and the resist etch selectivity versus chrome is not good. Tom said the industry will need a new e-beam resist that can survive the chrome etch process -- some thought it might be more productive to work on the absorber material, to abandon chrome and move to dense MoSi or TaN. Etch loading and etch uniformity problems are also significant challenges. If these errors are stable in the etch tools, they can be offset in the design. 
Inspection, repair and verification steps drive mask costs more than e-beam write time as the industry moves to higher resolution nodes, and Franklin Kalk from Toppan expects this trend to continue, though he finds inspection tools and repair tools are just keeping up with the challenge. The recent addition of good wafer plane inspection tools such as the Zeiss and Applied Materials AIM tools and new wafer plane simulation software from KLA-Tencor do sort out which defects will print, and can verify that repairs have been made correctly, but he is concerned that the very small market for these tools limits their availability. This is particularly true for EUV mask inspection and verification tools, though he said he was not yet ready to discuss this.

\section{EUV mask inspection: Not if, but when?}

Picking up the conversation on EUV masks, Ted Liang of Intel said that the Intel mask shop had all the tools that it needed to make working EUV masks except one -- Intel, and this industry, needs an EUV AIM tool. What's needed is a tool to help determine which defects really print -- but there is no supplier on the horizon. It is possible that the defect goal for EUV masks is tighter than it needs to be. Intel is also looking at ways to work around EUV mask defects; if mask blank defects can be mapped prior to e-beam exposure, it may be possible to shift or alter the pattern so that small defects will not print. An at-wavelength (13.5nm) AIM tool is needed to verify EUV masks are defect free before sending them to a production line. Liang reminded the audience that Intel is in $45 \mathrm{~nm}$ volume production now and will be moving to the $32 \mathrm{~nm}$ node next year, and the chipmaker wants EUV masks in CY2011 to be ready for production at the $16 \mathrm{~nm}$ node in CY2013.

Henry Yun of SEMATECH predicted that EUV masks will be over $40 \%$ of advanced mask work in 2013 or 2014 . He expects production using EUV masks will begin in earnest at the $22 \mathrm{~nm}$ node. Progress on EUV sources and tools looks good (beta units should be in IC maker pilot lines in CY2009). EUV resist has made impressive progress this year, now that alpha tools and the LBNL Synchrotron are available to run tests. Mask defects are the most serious challenge, and Henry described SEMATECH's work in this area. Pits in mask blanks cannot be repaired, so work continues on blank polishing methods. Also, particle inspection tools continue to be improved; LBNL is building an actinic particle scanning capability, which will not be a production tool, but will enable correlation of optical and actinic detection methods. Another iteration of improvements is being made to the multi-layer coating tools. The level of mask defects can be reduced as quickly as the detection technology is improved. In addition, some work-around ideas are now being considered. Henry would like to see mask blanks come with alignment marks so that defect location can be known and the mask pattern moved and adjusted to compensate for defects. He closed by reiterating the need for an actinic AIM tool for EUV mask verification -- the availability of which, he lamented, is limited by commercial issues, not technology ones. 


\section{Haze, 3D, DP, EUV, NIL}

Brian Grenon of Grenon Consulting described mask lifetime problems, with a map showing how haze formation on masks used in 193nm exposure tools is a global problem. As maskmakers have improved their cleaning process, it has become clear the problem is also a mask storage and lithography tool (fab environment) problem. And as maskmakers use more new materials such as MoSi, the chemical composition of haze changes to include these new materials, so the haze issue persists -- in fact, particles on masks now account for only about 33\% of mask "defects," Grenon reported. He pointed out that as the industry moves to EUV the issue of thin organic films will be an even greater problem -- at $13.5 \mathrm{~nm}$ any organic film will cause problems. Thus, mask "maintenance" costs will increase, he predicted, and the industry needs to consider this in their cost models as they look at the relative cost of different lithography choices.

Rick Silver of NIST reviewed CD and image placement metrology challenges, pointing out that the 3D shape of resist images is needed today, and 2D knowledge is insufficient. He believes optical scatterometry will be extendable through the $22 \mathrm{~nm}$ node for CD measurement. His team continues to improve ebeam modeling of SEM behavior, and is adding surface charging effects to improve these tools (AFM continues to be their reference tool).

Paul Ackmann of AMD noted scanner makers are going to reach their overlay goals for double patterning, and are now putting the focus on masks which will be key to DP. He made a case for including more image placement measurement points within each die, which will enable higher order correction for overlay errors. He also shared some cost analysis presented earlier at the conference, showing that EUV will be more cost-effective than double patterning at the $16 \mathrm{~nm}$ node.

Naoya Hayashi of DNP discussed making templates for nano-imprint lithography (NIL). Currently a $100 \mathrm{Kev}$ single spot e-beam tool is used to write NIL template patterns -- which are about $50 \times$ too slow for volume production. With the single spot e-beam tool, DNP can make $15 \mathrm{~nm}$ linespace patterns. With a $50 \mathrm{Kev}$ variable shaped beam (VSB) system, DNP has made very nice looking $24 \mathrm{~nm}$ linespace patterns and $28 \mathrm{~nm}$ dense contact and pillar patterns. Image placement errors are approximately $6 \mathrm{~nm} 3 \sigma$ over a mask area, and less over the $28 \mathrm{~mm} \times 24 \mathrm{~mm}$ area needed for a $1 \mathrm{X}$ template. Incremental improvements in the e-beam writer, inspection, and repair tools are needed to meet tolerances for the $32 \mathrm{~nm}$ node. Defects are an issue for 1X NIL templates.

\section{Q\&A: Cost concerns, EDA needs, NIL hurdles}

Questions during the audience participation time reflected a keen interest in challenges of making EUV masks. None of the panel members had discussed 
ways to manage EUV mask blank flatness and bending. Henry Yun noted that (a) the chuck will be made very flat so masks can be moved from tool to tool, and (b) electrostatic chucking will pull each mask flat. The residual unflatness and image placement errors due to mask bending will be modeled and corrected in the pattern data prior to pattern writing. (These issues had been covered in detail during on Thursday.)

Doug Resnick observed that defect data on EUV masks differed between the SEMATECH presentation and Intel presentation. Henry said his numbers reflect champion results and a scaling formula that SEMATECH uses to extrapolate EUV defects from measured optical detection tools. Ted Liang said he used average data for optically detected defects.

Is line-edge roughness (LER) an issue for masks? Ted Liang answered: Not really; the LER is not transmitted through scanner lenses. This is really a wafer level issue.

Greg Hughes asked if the IMS ion mask exposure Tool (iMET) could be used as a mask writer, and that is the plan; IMS presented their results on Tuesday. (By using multiple beams of hydrogen ions, not electrons, IMS can expose non-CAR resists at a sufficient rate; they showed excellent results at $16 \mathrm{~nm}$.) The IMS presentation included an announcement that IMS and NuFlare will establish a joint development project to build an ion multi-beam mask exposure tool.

There was an audience challenge regarding EUV mask cost expectations. Brian Grenon anticipates hydrocarbon contamination between multi-level coating layers may cause EUV mask yield and lifetime problems. Franklin Kalk expects EUV blank cost to go up, and EUV mask yield will drop. Franklin added that as the industry adopts double patterning added benefits will be discovered, making it more cost-effective for a longer time (which has been the history of similar advances). Kalk observed that each year the EUV crossover point moves out; SEMATECH's Yun agreed with the schedule slip, but added that he expected EUV blank cost to come down and yields to improve as volume increases (typical for a learning curve). Clearly this issue will be a hot topic at future BACUS meetings.

Warren Montgomery of SEMATECH observed that it was time for maskmakers to "share the pain" by adopting better resists. Brian Grenon added, "And new absorber materials" to improve the etch process and mask lifetime.

More back-and-forth from the audience participation segment of the program:

- AMD's Ackmann reminded everyone that equipment costs drive mask costs. Tools for writing, inspecting, repairing, and verifying are expensive and have a low throughput. 
- Was there a chance that the EDA task would get simpler with EUV? "No," replied Luigi Capotieci of AMD; EUV masks will need OPC and verification, which translates into lots of computing time. Liang of Intel observed that once maskmakers find a good trick (e.g., RET) they don't abandon it. Ideas presented earlier about defect mitigation (moving and altering patterns to cover up defects) would increase computing needs.

- Since a defect free mask seems impossible, how many defects might be tolerated? 100 defects may be OK, if they are small, Ted Liang answered.

- Why isn't NIL in production (for ICs)? Throughput is only at 4wph; overlay is at $15 \mathrm{~nm}$. Inspection and repair infrastructure is not ready. The risk of repeating defects on the production line also needs to be addressed. How can people monitor for such problems and remove a template for cleaning when a printing defect is observed?

From this panel discussion it seems maskmaking infrastructure for the $32 \mathrm{~nm}$ node is nearly ready for production, since there were few questions regarding this. The audience was much more interested in their next challenge, EUV masks. This is progress -- clearly this audience has accepted that EUV source and resist technologies are moving at the right pace toward production. As this group turns its attention to EUV maskmaking, it appears the IC industry will finally adopt EUV for some layers at the $22 \mathrm{~nm}$ node and for many more layers at the $16 \mathrm{~nm}$ node. This is Henry Yun's expectation. He may be right this time.

Griff Resor

\footnotetext{
* Reprinted from the October 21, 2008 edition of WaferNEWS. Copyright 2008 by PennWell. View other articles from Solid State Technology at: www.solid-state.com.
} 
Downloaded From: https://www.spiedigitallibrary.org/conference-proceedings-of-spie on 26 Apr 2023

Terms of Use: https://www.spiedigitallibrary.org/terms-of-use 